\title{
Bandwidth of Linearized Electrooptic Modulators
}

\author{
Uri V. Cummings and William B. Bridges, Life Fellow, IEEE, Fellow, OSA
}

\begin{abstract}
Many schemes have been proposed to make high dynamic range analog radio frequency (RF) photonic links by linearizing the transfer function of the link's modulator. This paper studies the degrading effects of finite transit time and optical and electrical velocity dispersion on such linearization schemes. It further demonstrates that much of the lost dynamic range in some modulators may be regained by segmenting and rephasing the $R F$ transmission line.
\end{abstract}

Index Terms-Bandwidth, electrooptic, linearized, modulators, photonic-link.

\section{INTRODUCTION}

$\mathbf{E}$ LECTROOPTIC intensity modulators have inherently nonlinear transfer functions which may limit the dynamic range of the photonic link through the production of harmonic and intermodulation distortion. Many schemes have been proposed to reduce the distortion byproducts of these modulators by linearizing their transfer functions; for example see the review paper by Bridges and Schaffner [1], and the references therein. The proposed applications for the resulting high dynamic range links include antenna remoting, photonic-coupled phased-array antennas, and cable television transmission.

Linearizing a modulator is a challenge. All of the proposed linearization schemes involve the cancellation of selected distortion terms, and this cancellation depends critically on modulator device parameters. Electrical biases very likely will require active control, and in some modulators, radio frequency (RF) or optical levels will require more accuracy than is realizable with current fabrication techniques.

For applications higher than $1 \mathrm{GHz}$, traveling wave electrode structures are mandatory to overcome the limitations resulting from interelectrode capacitance and finite transit time. A further difficulty with some popular electrooptic materials, such as lithium niobate, is that the electrical and optical waves travel at different velocities over the finite interaction length of the device, a result of material dispersion. This property limits the modulation-index $\times$ voltage product at high frequencies.

Given the critical dependence of the linearization scheme on modulator parameters, it is a fair question to ask, "How will velocity mismatch effect the linearization results?" This paper addresses that question for several popular modulator types. The summary result is that good velocity matching is essential to successfully linearize some but not all of the

Manuscript received October 24, 1997; revised April 21, 1998. This work was supported in part by the United States Air Force Rome Laboratories under Contract F30602-C-96-0020.

The authors are with the California Institute of Technology, Pasadena, CA 91125 USA.

Publisher Item Identifier S 0733-8724(98)05646-1.
TABLE I

The Parameters of a CANONICAL Optical LinK

\begin{tabular}{cccc}
\hline Velocity Mismatch & $\Delta \mathrm{n}$ & 1.8 & - \\
Mod. Length & $l_{m}$ & 10 & $\mathrm{~mm}$ \\
Laser Power & $\mathrm{P}_{L}$ & 100 & $\mathrm{~mW}$ \\
Laser Noise & $\mathrm{R}_{\mathrm{IN}}$ & -165 & $\mathrm{~dB} / \mathrm{Hz}$ \\
Optical Loss & $\mathrm{L}_{O}$ & -10 & $\mathrm{~dB}$ \\
Mod. Sensitivity & $\mathrm{V}_{\pi}, \mathrm{V}_{s}$ & 10 & $\mathrm{~V}$ \\
Mod Impedance & $\mathrm{R}_{M}$ & 50 & $\Omega$ \\
Det. Responsivity & $\eta \mathrm{D}$ & 0.7 & $\mathrm{~A} / \mathrm{W}$ \\
Det. Load & $\mathrm{R}_{D}$ & 50 & $\Omega$ \\
Noise Bandwidth & $\mathrm{BW}$ & 1 or $10^{6}$ & $\mathrm{~Hz}$ \\
\hline
\end{tabular}

modulators. The details differ significantly from one modulator type to another. This paper treats the frequency dependence of six modulator configurations: a standard Mach-Zehnder modulator (MZM), a dual parallel Mach-Zehnder modulator (DPMZM) and a dual series Mach-Zehnder modulator (DSMZM), a simple directional coupler modulator (DCM) at two different bias points, and a directional coupler modulator linearized with two additional dc biased directional couplers in series optically (DCM2P). The results of linearizing these modulators (except for the DSMZM) without regard for transit time were reported in [1]. Now, we report the comparisons including transit time and velocity mismatch.

\section{LINK MODEL}

This paper assumes the same simplified photonic link and parameters as [1], but now adds the parameters for the finite frequency calculations. They are the effective index mismatch $\Delta n$, the modulator length $l_{m}$, and the frequency $f_{0}$. The link consists of a laser, an electrooptic modulator, a length of fiber, and a detector. The model excludes electronic preamplification and postamplification. Table I shows all of the parameters associated with this link. These parameters are assumed to have no frequency dependence in the model. The parameters for the active length and the velocity mismatch are typical for $\mathrm{LiNbO}_{3}$ modulators using simple parallel strip electrodes with no velocity matching. That is, $n_{\text {microwave }} \approx 4.0$ and $n_{\text {optical }} \approx 2.2$, and thus $\Delta n \approx 1.8$. Velocity matching will result in a lower value of $\Delta n$.

As in [2], the results are calculated numerically, since no closed-form solution exists for the transfer function of some modulator types. A program, written in $\mathrm{C}$, calculates the frequency-dependent gain and dynamic range. A two-tone electrical test signal, with frequencies $f_{1}$ and $f_{2}$ drives the modulator. The Fourier transform of the output is evaluated to 
find the gain, the harmonic content, and the intermodulation content of the link.

Let $P\left(p_{\text {in }}, t\right)$ be the electrical signal power after the detector, given the modulator RF drive power $p_{\text {in }}$. Let $\tilde{P}\left(p_{\text {in }}, f\right)$ be the Fourier transform of $P\left(p_{\mathbf{i n}}, t\right)$.

The gain is

$$
\operatorname{Gain}_{\mathrm{dB}}=10\left\{\log \left[\tilde{P}\left(p_{\mathbf{i n}}, f_{1}\right)\right]-\log \left(p_{\text {in }}\right)\right\}
$$

The small signal gain is obtained by evaluating (1) at sufficiently small $p_{\text {in }}$ that the $\log -\log$ plot of $\tilde{P}\left(p_{\text {in }}, f_{1}\right)$ is linear with slope one. In practice, a good value of $p_{\text {in }}$ for this calculation is the geometric mean of $p_{\text {sat }}$ (the power that drives the modulator voltage to about $V_{\pi}$ ) and the precision of double precision floating point numbers, or about $p_{\text {in }}=-100 \mathrm{dBm}$.

The spur free dynamic range, $\mathrm{DR}_{\mathrm{dB}}$, is the power interval that spans the input power level at which the signal is just distinguishable from the noise and the input power level at which the strongest distortion term becomes distinguishable from the noise. The calculation of $\mathrm{DR}_{\mathrm{dB}}$ is

$$
\begin{aligned}
R_{\mathrm{dB}}\left(p_{\text {in }}\right)= & 10 \max \\
& \left\{\log \left[\tilde{P}\left(p_{\text {in }}, 2 f_{1}-f_{2}\right)\right]\right. \\
& \left.\log \left[\tilde{P}\left(p_{\text {in }}, 2 f_{1}\right)\right]\right\}- \text { noise }_{\mathrm{dB}} \\
p_{0}= & \min \left(\left.p_{\text {in }}\right|_{R_{\mathrm{dB}}\left(p_{\text {in }}\right)=0}\right) \\
\operatorname{DR}_{\mathrm{dB}}= & 10 \log \left[\tilde{P}\left(p_{0}, f_{1}\right)\right]-\text { noise }_{\mathrm{dB}} .
\end{aligned}
$$

$R_{\mathrm{dB}}\left(p_{\mathrm{in}}\right)$ is the maximum of the relevant distortion terms minus the noise level in $\mathrm{dB}$. Of the roots of $R_{\mathrm{dB}}\left(p_{\mathrm{in}}\right), p_{0}$ is the root that occurs at the lowest power level. $\mathrm{DR}_{\mathrm{dB}}$ is the difference between $p_{0}$ and the input power level at which the signal intersects the noise floor. Since the log-log plot of the signal has slope one, this interval is equivalent to the signal power minus the noise power at the RF drive power at which the distortion power equals the noise level which is (4). Fig. 1 describes the dynamic range calculation for a simple Mach-Zehnder modulator. While dynamic range generally refers to all harmonics and intermodulation products, in practice, there are two dominant distortion terms, the second harmonic, $\tilde{P}\left(p_{\text {in }}, 2 f_{0}\right)$, and the first intermodulation product, $\tilde{P}\left(p_{\text {in }}, 2 f_{0}-f_{1}\right)$. The linearized modulators in this paper are separated into two categories, each with a different definition of dynamic range. Equation (2) applies to broadband, or superoctave modulators. That is, $R_{\mathrm{dB}}\left(p_{\mathrm{in}}\right)$ is the maximum of the second harmonic and the intermodulation product. $R_{\mathrm{dB}}\left(p_{\mathrm{in}}\right)$ is defined differently in narrow band or suboctave modulators. In this second category, $R_{\mathrm{dB}}\left(p_{\mathrm{in}}\right)$ equals only the third order intermodulation product. In ordinary modulators, the $\log -\log$ plots of the distortion terms are linear intersecting the noise floor only once. In linearized modulators, the distortion terms are nulled at discrete power levels. They may cross the noise level more than once. It is then necessary to find all of the roots of the distortion minus the noise, $R_{\mathrm{dB}}$, and take the root representing the lowest RF drive power in the definition of dynamic range.

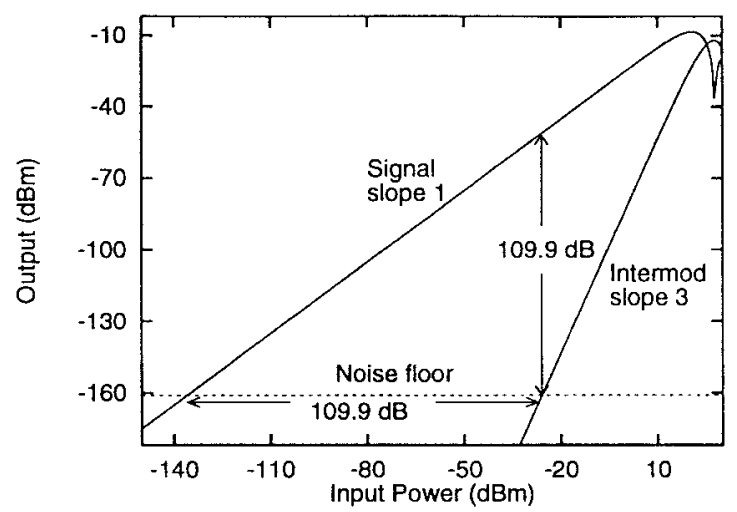

Fig. 1. The signal and third-order intermodulation product of a simple Mach-Zehnder modulator. Since there is effectively no second-harmonic distortion, $R_{\mathrm{dB}}$ equals the intermodulation product. $R_{\mathrm{dB}}$ crosses the noise floor only once at an input power level of $p_{0}=-26 \mathrm{dBm}$. Since the signal has slope one, the dynamic range may be found from the difference between the signal and $p_{0}$ either horizontally or vertically in the plot, giving the familiar dynamic range triangle.

\section{A. Computation of Modulators with Velocity Dispersion}

The frequency dependent output of any modulator with a known dc transfer function is calculable. Farwell gives a detailed computational method for this in [2]. The mathematics are straightforward. Let $A_{\text {in }}(t)$ and $A_{\text {out }}(t)$ be the input and output complex amplitudes of the optical wave in a modulator. Let $H\left(V, l_{m}\right)$ be the transfer function, where $V$ is the normalized modulator drive voltage and $l_{m}$ is the active length of the modulator. If the optical and electrical waves travel at the same velocity, or if the operating frequency is so low that $V$ is effectively constant over $l_{m}$, then the output is given by

$$
A_{\text {out }}(t)=H\left[V(t), l_{m}\right] A_{\text {in }}(t) .
$$

Even if there is significant velocity dispersion while the two waves travel the distance $l_{m}$, over a short enough section of the guide $(\Delta x)$, the change in the complex optical amplitudes may be described with the dc transfer function. This is the basis for the frequency dependent calculation. Let $x$ be the coordinate along the optical waveguide, and let $t$ be time. Then

$$
\begin{aligned}
& \lim _{\Delta x \rightarrow 0} A(x-\Delta x, t-\Delta t) H[V(x-\Delta x, t-\Delta t), \Delta x] \\
& \quad \rightarrow A(x, t) .
\end{aligned}
$$

The optical and electrical signals are now functions of two variables, $x$ and $t$. In (6) the elapsed time equals the incremental length divided by the electrical velocity $\Delta t=\Delta x / \nu$.

Let there be $N$ equally spaced increments of $x$ and $M$ equally spaced increments of $t$, and let $x_{0}=0$ and $x_{N}=l_{m}$. The modulator is divided into $N$ sections over which the optical and electrical fields are approximately constant. The finite product of the resulting $N$ unitary dc transfer matrices gives the overall transfer function from $A_{\text {in }}(t)$ to $A_{\text {out }}(t)$. Let $A\left(x_{0}, t\right)=A_{\text {in }}(t)$, and $A\left(x_{N}, t\right)=A_{\text {out }}(t)$, and note that $A_{\text {out }}(t)=0$ for $t<x_{N} / \nu$. The approximate modulator 
transfer function is

$$
A_{\text {out }}\left(t_{j}\right) \approx\left\{\prod_{k=0}^{N-1} H\left[V\left(x_{k}, t_{j}-\frac{x_{N-1-k}}{\nu}\right), \frac{l_{m}}{N}\right]\right\} A_{\text {in }}(t) .
$$

The function $V(x, t)$ representing the two tone test is

$$
\begin{aligned}
V\left(x_{k}, t_{j}\right)= & p_{\text {in }}\left[\cos \left(\frac{j}{M}-\frac{i}{N} \cdot \frac{\gamma}{f_{1}}\right)\right. \\
& \left.+\cos \left(\frac{j}{M}-\frac{i}{N} \cdot \frac{\gamma}{f_{2}}\right)\right] \\
\gamma \equiv & \frac{f_{0} \Delta n l_{m}}{c} .
\end{aligned}
$$

The parameter $\gamma$ depends on the operating frequency $f_{0}$, the difference between the optical and electrical indices, $\Delta n$, the active length $l_{m}$, and the velocity of light $c$. It is important to note that the calculation results depend solely on $\gamma$ and not on $\Delta n, l_{m}$, and $f_{0}$ independently. Thus the results of different lengths or relative wave velocities at different frequencies will be the same if $\gamma$ is the same. The curves shown below are universal in the sense that they apply to more than the "worst case" velocity mismatch, which is $\Delta n=1.8$ for lithium niobate modulators. Any change in $\Delta n$ or $l_{m}$ leads to a rescaling of the frequency axis for the gain and dynamic range plots.

Equation (7) is general and is the basis for the frequency dependent computations in the model. However, when the active region of a modulator consists of only simple phase shifts, as it does for the Mach-Zehnder modulator, a further simplification may be made. The transfer function is just a diagonal matrix of exponentials. Instead of multiplying exponentials, their arguments are summed. This is equivalent to integrating the location variable out of the voltage function. That is

$$
\begin{aligned}
A_{\mathrm{out}}(t) & =H\left[\int_{0}^{l_{m}} V\left(x, t-\frac{l_{m}-x}{\nu}\right) d x, l_{m}\right] A_{\text {in }}(t) \\
A_{\mathrm{out}}\left(t_{j}\right) & \approx H\left[\sum_{k=0}^{N-1} V\left(x_{k}, t_{j}-\frac{x_{N-1-k}}{\nu}\right), l_{m}\right] A_{\text {in }}(t) .
\end{aligned}
$$

The approximation introduced in (11) comes from the substitution of a summation for an integral, the relationship between $A_{\text {out }}$ and $H$ shown in (10) is exact. It may seem odd to use an approximation for a function for which a trivial analytic solution exists [integral of (8)]. However, this is done to mirror the calculation technique for directional couplers and to support the modeling of modulator voltage functions which may not have an analytic integral representation.

In the C-program mentioned above, the temporal increments, $M$ are restricted to be powers of two, so that a radix-2 fast Fourier transform (FFT) algorithm may be used for the spectral analysis. ${ }^{1}$ Fig. 2 shows the convergence of the gain of a Mach-Zehnder modulator as a function of spatial increments $N$ at 5,10, 20, and $40 \mathrm{GHz}$. The error is normalized, and

\footnotetext{
${ }^{1}$ It is customary to use the FFT algorithm which is $O(N \log (N))$ instead of the DFT algorithm which is $O\left(N^{2}\right)$. However, it should be noted that the algorithm to compute the modulator output is $O\left(N^{2}\right)$, so the time spent in the FFT algorithm is inconsequential.
}

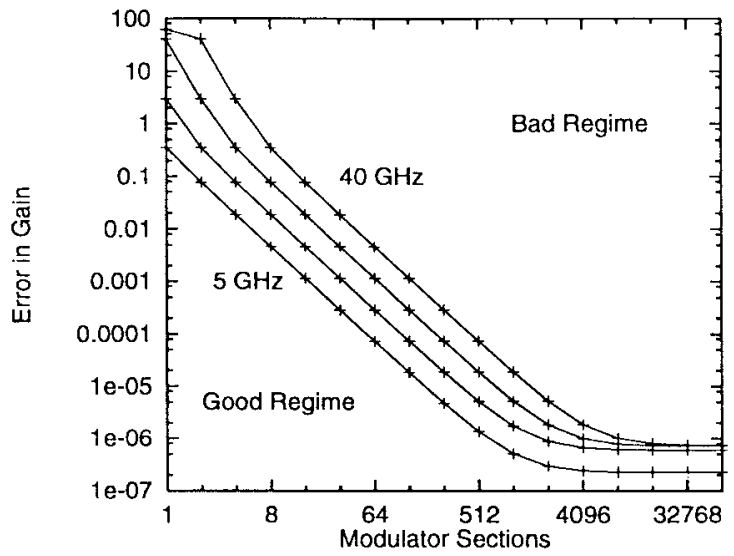

Fig. 2. The convergence of the calculation of the gain of a Mach-Zehnder modulator (MZM), with an increasing number of modulator sections. The error is the magnitude of the calculated gain (not in $\mathrm{dB}$ ) minus the analytical value normalized by the dc analytical value for the gain. The curves of the $\log -\log$ plot are for 5, 10, 20, and $40 \mathrm{GHz}$.

thus the $y$-axis value "one" corresponds to a $3 \mathrm{~dB}$ error and " 0.1 " corresponds to a $0.4 \mathrm{~dB}$ error in the calculated gain. The curves in Fig. 2 are linear until the error is six-to-seven orders of magnitude below the dc value of the gain (since the plot is $\log -\log$, constant slopes do not indicate geometric convergence). The convergence saturates at a normalized error of about $10^{-6}$ because the RF drive power for the small signal gain calculation was arbitrarily chosen to be -100 $\mathrm{dBm}$. Whether in the calculation for gain or dynamic range, the numbers have components that differ by $6-7$ orders of magnitude. Since these components occupy the same mantissa, there is a loss in accuracy not recovered by the floating decimal point. Double precision numbers must be used to attain a satisfactory accuracy. It is interesting to note that the calculated points form a horizontal line across the curves in the linear regime. This indicates that doubling $\gamma$ (by doubling the frequency for instance) exactly requires a doubling of the modulator sections to achieve the same error. Efficient code allows the calculation hundreds of frequency points with a 128-point FFT and a comparable number of spatial increments in seconds on a contemporary desk-top machine $(120 \mathrm{MHz}$ Pentium processor).

\section{MACH-ZEHNDER AND DiRECTIONAL COUPLER MODUlatorS}

The most common electrooptic modulator is the Mach-Zehnder interferometer (MZM). It has a sine-squared transfer function, and the gain is a sinc function of the frequency-length-index product, $\gamma$. When biased at the half-wave voltage, $V=0.5 V_{\pi}$, it attains its maximum linearity and dynamic range. All even-order harmonics are identically zero. The intermodulation distortion product solely determines the dynamic range, even in a super-octave system. The dynamic range is independent of frequency; the signal decays with frequency, but the intermodulation product decays identically. Thus, the range of RF drive powers (in $\mathrm{dB}$ ), over which their are no spurs above the noise, shifts with a change in frequency, but it does not expand or 


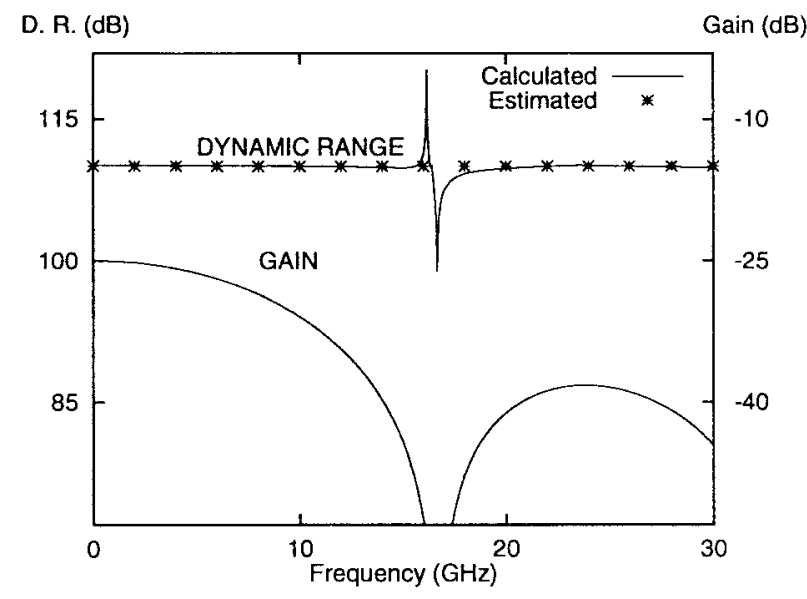

Fig. 3. Gain and dynamic range of a standard Mach-Zehnder modulator. The pole and zero in the dynamic range are the frequencies at which the signal and intermodulation products go to zero, respectively.

contract. Given its analytical simplicity and widespread use, the Mach-Zehnder is used first to evaluate the accuracy of the numerical calculation, and then it is used for a comparison to the linearized modulators.

Fig. 3 shows the calculations for gain and dynamic range of a simple MZM. The gain has the form $[\sin (\pi \gamma) /(\pi \gamma)]^{2}$ with zeros at multiples of $16.2 \mathrm{GHz}$ (where $\gamma=1$ for the canonical parameters from Table I), and a low frequency link gain of $-25.5 \mathrm{~dB}$ (also appropriate for the link parameters). The dynamic range is flat except for a null and singularity near the gain null. This is a simple numerical artifact, resulting from the finite frequency difference between the two tones in the driving function. The signal, $f_{1}$, and the intermodulation product, $2 f_{1}-f_{2}$, are at slightly different frequencies, and hence, they null at different frequencies. The dynamic range goes to zero at the signal null and it goes to infinity at the distortion null.

Fig. 4 shows the analogous calculations for a directional coupler modulator. The low frequency gain is $-24.8 \mathrm{~dB}, 0.7$ $\mathrm{dB}$ better than Mach-Zehnder. ${ }^{2}$ The first null of the gain of the directional coupler occurs at $26 \mathrm{GHz}, \gamma=1.6$, compared to $16 \mathrm{GHz}$ for the MZM. The first lobe of the gain curve does not correspond to the sinc function of the Mach-Zehnder. However, subsequent nulls are periodic with a $16 \mathrm{GHz}$ period, resulting from the underlying $\gamma$ of the directional coupler. The frequency at which the gain has fallen by $3 \mathrm{~dB}$ is $40 \%$ higher than that of the Mach-Zehnder modulator with the same index-length product.

The dynamic range compares unfavorably to that of the Mach-Zehnder. At low frequency it is similar to that of the MZM, and it is approximately flat with frequency. However there is a kink in the curve at $1.8 \mathrm{GHz}$ (left vertical arrow), after which the dynamic range decays rapidly with frequency. Unlike the Mach-Zehnder, where all even-order derivatives of the transfer function are identically zero when the modulator

\footnotetext{
${ }^{2}$ The comparison of the gain bandwidth product between the MZM and the DCM assumes that $V_{\pi}=V_{s}$; that is, the modulators have the same normalization voltages. While these voltages should be similar in the same manufacturing process, it is hard to directly compare them since the electrode geometries and crystal orientations of the two modulator types may differ.
}

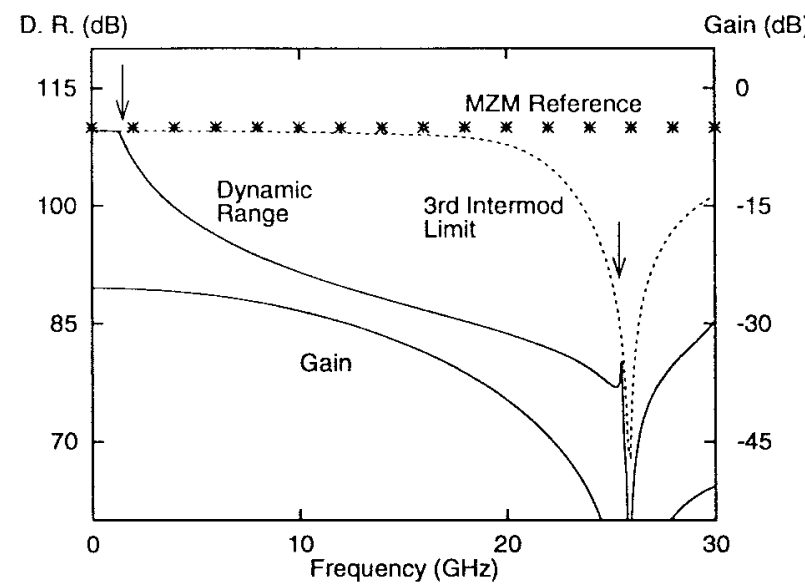

Fig. 4. Gain and dynamic range of a directional coupler modulator biased at the $0.43 V_{s}$ point. The two arrors indicate that the dynamic range changes from intermodulation-limited to second-harmonic limited or vice versa. If the second harmonic is ignored (suboctave operation), the dynamic range follows the dashed curve.

is biased at $0.5 V_{\pi}$, in the directional coupler only the second derivative is zero at its optimal bias, $0.43 V_{s}$. Distortion migrates from nonzero fourth-, sixth-, eighth-, etc., order derivatives to the frequency at which the second harmonic occurs. It does so rapidly with increasing frequency, and it equals the third order intermodulation product at $1.8 \mathrm{GHz}$. At higher frequencies, distortion from these nonzero even derivatives limits the dynamic range. The intermodulationlimited dynamic range (as if this were a suboctave modulator) is shown with a dotted line to demonstrate the intermingling of the second harmonic and third order intermodulation product. The bias voltage that nulls the total second harmonic, which arises from all even order derivatives, is thus a function of frequency in the DCM, but it is independent of frequency in the MZM. At any finite frequency, there is a bias value which nulls the total second harmonic, but the null will hold only over a narrow bandwidth. While the gain compares favorably to the MZM, the dynamic range makes the directional coupler inferior to the Mach-Zehnder for superoctave, high-frequency applications.

\section{BROAD-BAND LINEARIZED MODULATORS}

Dynamic range values are calculated for two linearized broad-band, or superoctave, modulators: the dual parallel Mach-Zehnder (DPMZM) and the directional coupler with two passive sections (DCM2P) which are described in [4] and [5], respectively. The DPMZM has two identical, single Mach-Zehnder modulators in parallel optically and electrically but with unequal levels of optical and RF power driving the two modulators. Both modulators are biased at the $0.5 V_{\pi}$ point, but with opposite slopes, so the modulators are 180 degrees out of phase. Most of the optical power and a small fraction of the RF power drive one modulator. A small amount of optical power and the majority of the RF power drive the other modulator, creating relatively larger distortion products than in the first modulator. The two signals are combined incoherently in the photodetector. The RF and optical splits are adjusted so that the distortion terms cancel exactly, but the 


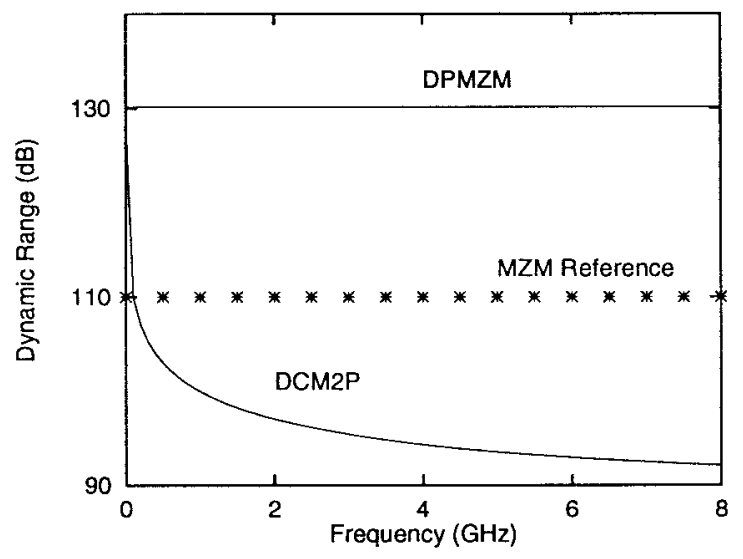

Fig. 5. Dynamic range comparison of two linearized modulators: a dual parallel Mach-Zehnder modulator (DPMZM) and a directional coupler modulator with two passive sections (DCM2P). As in Fig. 3 for the simple MZM the dynamic range of the DPMZM is independent of frequency. However, the dynamic range of the DCM2P drops precipitously with frequency.

signals do not. Since these two paths are in parallel, the effects of velocity mismatch apply equally to each Mach-Zehnder. Thus, the distortion terms still precisely cancel regardless of frequency, and this is the result shown in Fig. 5. While the DPMZM is robust to velocity mismatch, in practice it is hard to make broadband. A precise RF split must be maintained over the desired frequency band. If it varies, the dynamic range will decrease at all but the narrow frequency at which the RF split is optimized.

The linearized directional coupler with two passive sections can be adjusted to provide high dynamic range with both intermodulation and second harmonic reduction in the absence of velocity mismatch. However, it suffers severely from velocity mismatch as shown in Figs. 5 and 6. By $80 \mathrm{MHz}$ it is no better than the ordinary Mach-Zehnder and by $8 \mathrm{GHz}$ it is $18 \mathrm{~dB}$ worse than the ordinary MZM. Fig. 6 shows the first $50 \mathrm{MHz}$ of the DCM2P dynamic range in more detail. Unlike the DPMZM, in the DCM2P the mismatch between the RF drive and the modulated signal upsets the critical distortion cancellation conditions.

It has previously been reported [1] that the distortion cancellation condition is critically sensitive to the modulator parameters, particularly bias voltage. The voltage on the bias electrodes must be maintained to a very high accuracy. The accuracy required depends on the operating bandwidth, also explained in [1]. This requires active bias stabilization. Given the critical bias conditions and the fact that the distortion cancellation sections are in series, unlike the DPMZM, the rapid degradation with dynamic range is reasonable. The original experiments on this modulator were performed at audio frequencies where these effects would not be noticed [5]. Subsequently, measurements at 1 and $2 \mathrm{GHz}$ [6] were singlefrequency measurements, with the bias values reoptimized for the operating frequency; no bandwidth measurements around 1 and $2 \mathrm{GHz}$ were made.

\section{Suboctave Linearized Modulators}

For suboctave applications, the second harmonic may be ignored; the third-order intermodulation product alone de-

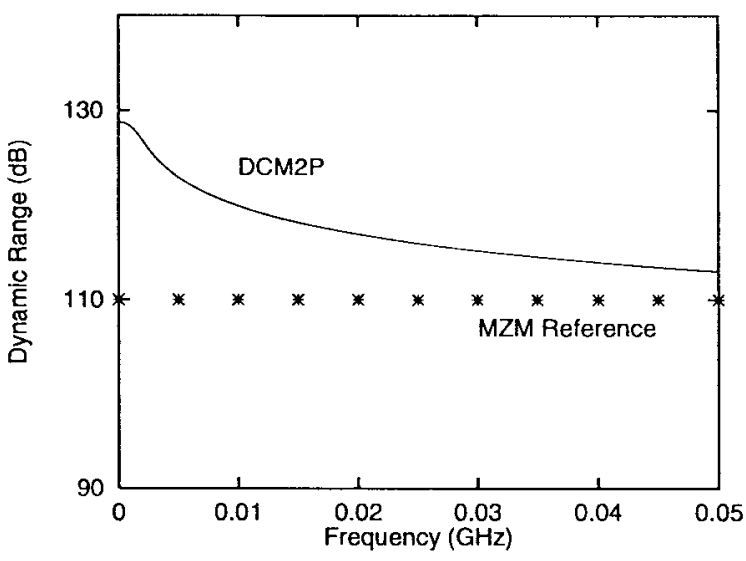

Fig. 6. Expanded plot of the dynamic range of the DCM2P shown in Fig. 5.

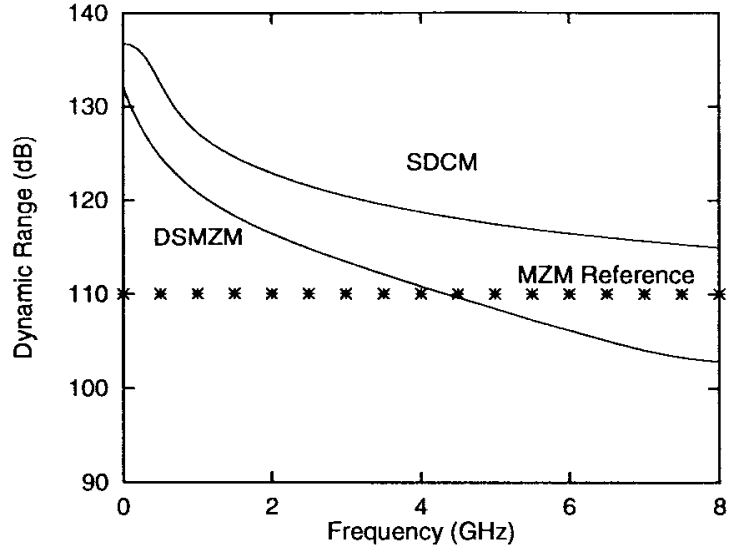

Fig. 7. Dynamic range comparison of two suboctave linearized modulators: a dual series Mach-Zehnder modulator (DSMZM) and a directional coupler modulator biased at $0.79 V_{s}$ (SDCM).

termines the dynamic range. Two suboctave modulators are analyzed in this section.

1) The dual series Mach-Zehnder modulator (DSMZM), as described in [7], which has two MZM's in series optically, the same bias voltage on each pair of electrodes, and a single RF electrode covering both modulators. ${ }^{3}$

2) A suboctave directional coupler modulator (SDCM), i.e., a directional coupler biased at $0.79 \mathrm{~V}_{s}$ istead of $0.43 \mathrm{~V}_{s}$ as described in [1]. Unlike the MZM, in the directional coupler, the intermodulation product nulls at a different voltage $\left(0.79 V_{s}\right)$ than the signal $\left(1.0 V_{s}\right)$. Thus no extra electrode sections (as in the DCM2P) are needed to make a suboctave directional coupler.

Fig. 7 shows the dynamic range as a function of frequency for the DSMZM and SDCM compared to the standard MZM reference (horizontal dotted line). Both of these modulators suffer from the effects of velocity mismatch and transit time. However, unlike the DCM2P, the DSMZM shows an advan-

\footnotetext{
${ }^{3}$ There are other cascaded Mach-Zehnders proposed in the literature. In some there are different bias voltages on the two electrodes. In [8] there is a time delay between the first and second modulator so that the RF drive and the modulated signal are rephased at the second Mach-Zehnder. While this version may be more common in the literature, for the purposes of a fair comparison, this modulation scheme is addressed in the section on periodic rephasing below. Additionally, in [9] a mixed directional coupler and Mach-Zehnder scheme purports to minimize the second and third harmonic.
} 


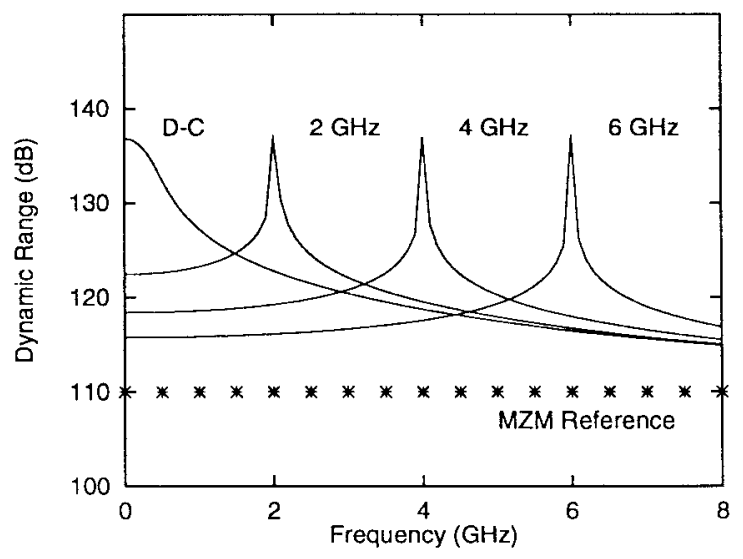

Fig. 8. Reoptmization of the suboctave linearized directional coupler modulator (SDCM). Slightly adjusting the bias voltage around $0.79 V_{s}$ fully recovers the low-frequency dynamic range optimum, but only over a narrow bandwidth.

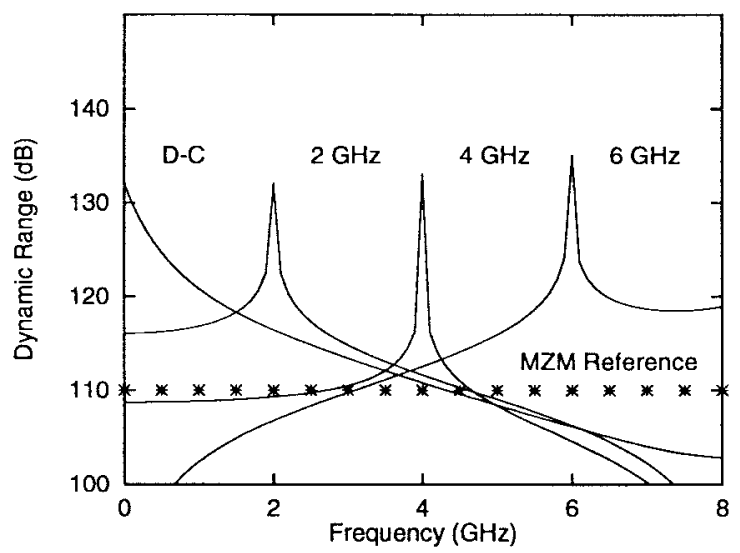

Fig. 9. Reoptimization of the dual series Mach-Zehnder modulator (DSM-ZM). Adjusting the bias voltage fully recovers the low-frequency dynamic range optimum, but only over a narrow bandwidth.

tage up to $4 \mathrm{GHz}$ over the standard Mach-Zehnder, and the SDCM is better still. The SDCM is consistently about $5 \mathrm{~dB}$ better than the DSMZM. At the SDCM bias $\left(0.79 V_{s}\right)$, the dc optical output is quite small, thus reducing the shot noise at the detector, which is the dominant term in the total noise (as described in [1]).

The bias voltage of the SDCM controls the frequency of the dynamic range optimum. Small adjustments in the bias around the dc value of $0.79 \mathrm{~V}$ allow the full recovery of the lowfrequency dynamic range optimum at any center frequency. Unfortunately, this results in a relatively narrow operating bandwidth around the reoptimization frequency, as shown in Figs. 8 and 9. The critical control of bias voltage will doubtless require the use of pilot tones to minimize the harmonic or intermodulation products. However, the results of Figs. 8 and 9 indicate that these pilot tones will have to be within the band of interest, not at low frequencies.

\section{THE EFFECTS OF NOISE}

Noise effects linearized modulators somewhat differently than standard modulators. In a standard MZM, the noise bandwidth reduces the dynamic range by $(\mathrm{BW})^{2 / 3}$. In Fig. 1 the signal is a line with slope one, and the third-order intermodu-

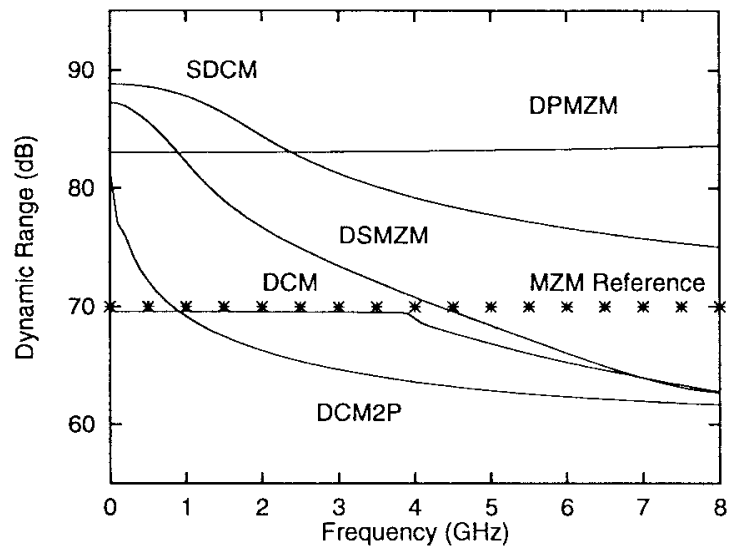

Fig. 10. Dynamic range comparison with $1 \mathrm{MHz}$ noise bandwidth of five modulator configuration: the superoctave dual parallel Mach-Zehnder modulator (DPMZM), the superoctave directional coupler modulator linearized by two passive couplers in series (DCM2P), the suboctave dual series Mach Zehnder (DSMZM), the suboctave linearized directional coupler (SDCM), and the standard directional coupler modulator (DCM).

lation product is a line with slope three. The "noise floor" is a third, horizontal line which forms a triangle with the signal and the intermodulation line. The length of the base of this triangle is the dynamic range (in $\mathrm{dB}$ ). The vertical position of the noise line is proportional to the $\log (\mathrm{BW})$, so from simple geometry, it is clear that the dynamic range goes as $(\mathrm{BW})^{2 / 3}$. However, in a linearized modulator the third-order intermodulation product at $\left(2 f_{2}-f_{1}\right)$ has been nulled. The dominant intermodulation term is at $\left(3 f_{2}-2 f_{1}\right)$, and it grows as the fifth power of the RF drive. Thus the noise bandwidth reduces the dynamic range by $(\mathrm{BW})^{4 / 5}$ (More complicated linearization schemes can result in even steeper slopes for intermodulation, as discussed in [1]). The excess dynamic range, $X$, of a linearized modulator over a ordinary modulator is

$$
X=C-\frac{4}{3} \log (\mathrm{BW})
$$

where BW is a the bandwidth in Hertz, and $C$ is a constant in $\mathrm{dB}$ equal to the difference between the dynamic range of a linearized modulator and a standard modulator with a $1-\mathrm{Hz}$ noise bandwidth. This equation is only valid in the approximation that the signal and intermodulation curves are straight lines. For a comparison of the suboctave DCM versus the standard MZM, $C$ equals $26 \mathrm{~dB}$. For a $1 \mathrm{MHz}$ noise bandwidth, $X=18 \mathrm{~dB}$. The dynamic range is often given for a $1-\mathrm{Hz}$ noise bandwidth, and this simple scaling rule is applied to find the dynamic range of a system with a realistic bandwidth.

Apart from the different scaling rules, the noise bandwidth has an additional effect on linearized modulators. The dynamic range becomes less sensitive to device variations, either bias voltages or fabrication parameters, as the noise bandwidth increases. This makes it easier to maintain the distortion cancellation than is implied by some of the previous figures. Fig. 10 shows the dynamic range for the four modulators discussed above, but now with a 1-MHz noise bandwidth. The DPMZM and the MZM reference lines are flat as in the $1-\mathrm{Hz}$ bandwidth case. The simple DCM is flat out to the 


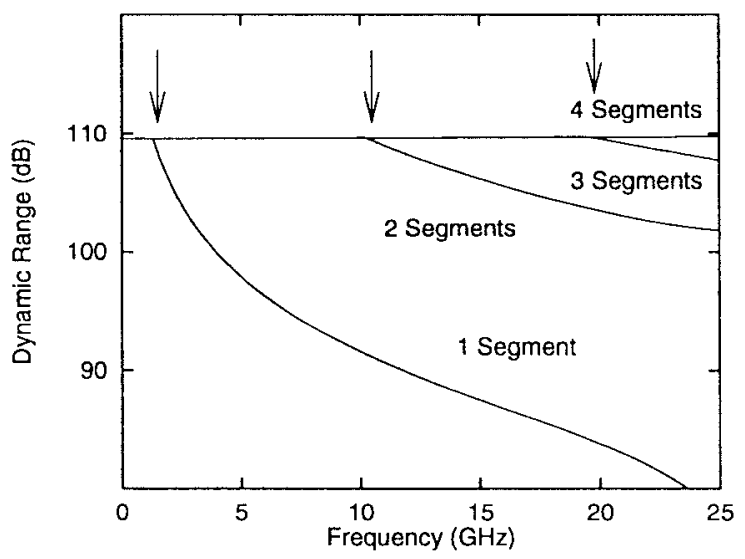

Fig. 11. Dynamic range versus frequency for the standard directional coupler modulator (DCM) with multiple electrode segments. The arrows show the breaks between the intermodulation and the second-harmonic limitation of the dynamic range.

frequency where the second harmonic exceeds the intermodulation distortion, as in Fig. 3. However, the crossover now occurs at $3.8 \mathrm{GHz}$ instead of $1.8 \mathrm{GHz}$. The DSMZM, SDCM, and DCM2P dynamic ranges, which all roll off with frequency, now do so at a slower rate than in the $1-\mathrm{Hz}$ bandwidth case. While the DCM2P still rolls off too quickly to be a useful modulator, the suboctave modulators are starting to show reasonable frequency performance. A similar reduction in peak dynamic range, with a broadening of the bandwidth over which it occurs, is also obtained in the bias reoptimized curves of Figs. 8 and 9.

\section{BANDWIDTh Recovery Through PERIODIC REPHASING}

One method of overcoming the degradation in dynamic range due to velocity mismatch is to break the transmission line into a number of segments and rephase the signal at the beginning of each segment. This is velocity-matching "on the average." The technique has been used successfully in a number of forms, for example, [10]-[12]. The program written for this study is easily modified to make calculations for such periodically rephased modulators, since the modulator is already broken up into a cascade of matrices. Thus, the modulator is incrementally velocity mismatched for a few matrices and is then rephased for the next section, and so on. Fig. 11 shows the results of such a calculation for the simple DCM link with the parameters used above, but with the modulator's transmission line having 1, 2, 3, and 4 segments. The 1-segment curve repeats the result in Fig. 4 (no rephasing) for reference. With only two segments (one rephasing), the bandwidth over which the dynamic range is flat improves vastly, and using four segments gives an essentially flat dynamic range. Of course, it would still be preferable to use a standard MZM for broadband links since it does not require rephasing.

A similar dramatic improvement is obtained in the SDCM, biased at the $0.79 V_{s}$ point, as shown in Fig. 12. The curve from Fig. 7 is shown for reference along with curves for two-, three-, and four-electrode segments. The curve for one segment initially shows a deep roll-off in dynamic range and then a more gradual roll-off, with only $5 \mathrm{~dB}$ of dynamic range improvement over the standard MZM remaining at $8 \mathrm{GHz}$.

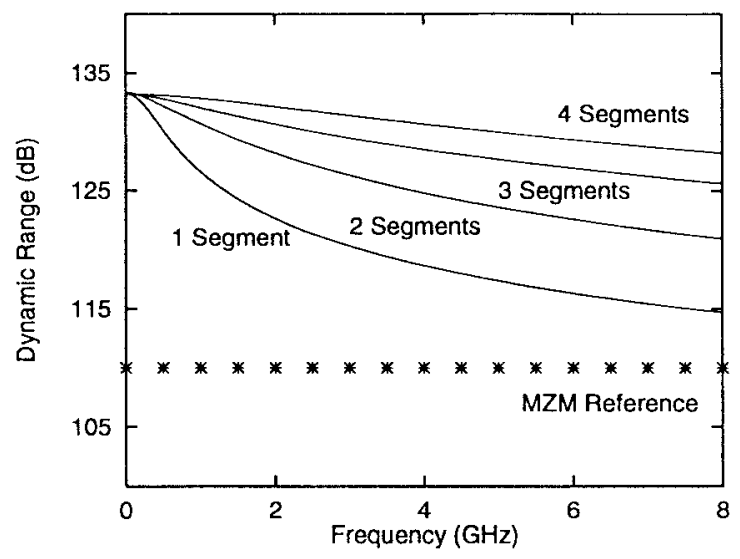

Fig. 12. Dynamic range versus frequency for the suboctave linearized directional coupler (SDCM) with multiple electrode segments.

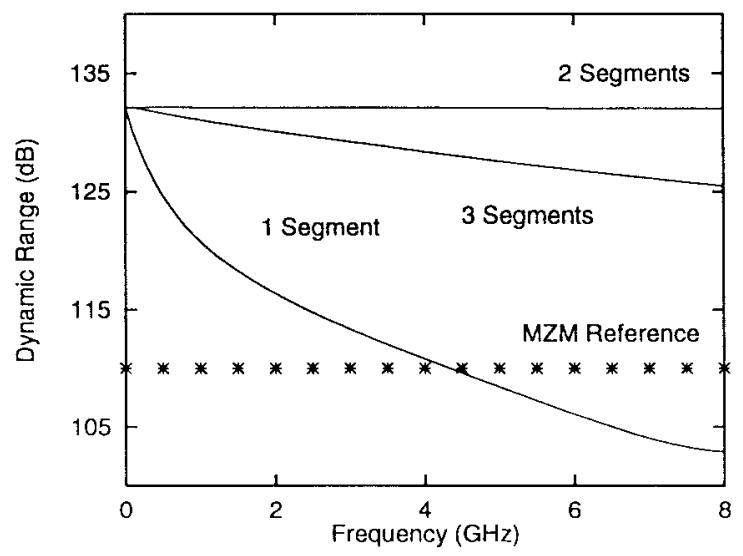

Fig. 13. Dynamic range versus frequency for the the suboctave dual series Mach-Zehnder (DSMZM) with multiple electrode segments. Note that two (and any even number) segment give frequency independent dynamic range.

With just two segments, the roll-off is made gradual over the whole range. With four segments, there is $18 \mathrm{~dB}$ of dynamic range improvement remaining at $8 \mathrm{GHz}$. This figure assumes a $1-\mathrm{Hz}$ noise bandwidth. When a $1-\mathrm{MHz}$ noise bandwidth is used, the roll-off is more gradual. For instance, the dynamic range of the two-electrode segment SDCM is better than the two-electrode DSMZM (shown in Fig. 13) up to $5.9 \mathrm{GHz}$.

A very interesting result of the application of rephasing is seen in the dual series Mach-Zehnder modulator. This modulator has been shown to give very high dynamic range values based on the intermodulation distortion, although it has, like the SDCM, a very large second harmonic content, restricting it to suboctave applications as stated in [8]. The decrease in dynamic range with frequency is shown in Fig. 13 in the curve labeled " 1 segment," meaning one set of traveling wave electrodes spanning two Mach-Zehnder modulators (with an admittedly unrealistic zero distance assumed between the two modulators). This is simply a repeat of the curve in Fig. 7. The surprise is that with only one rephasing (two segments) the modulator exhibits frequency-independent performance. Breaking the electrodes into three segments yields an improvement over one segment, but not as good as two or any even number of segments, all of which yield perfect performance. This behavior results from the symmetry of the modulators and their bias points. 


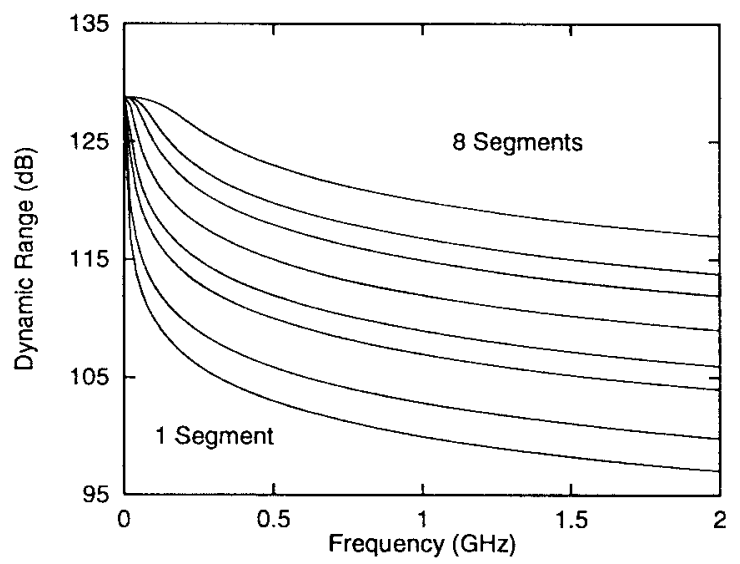

Fig. 14. Dynamic range versus frequency for the superoctave linearized directional coupler (DCM2P) with one to eight electrode segments. While rephasing yields improvements, the results remain inferior to other modulators.

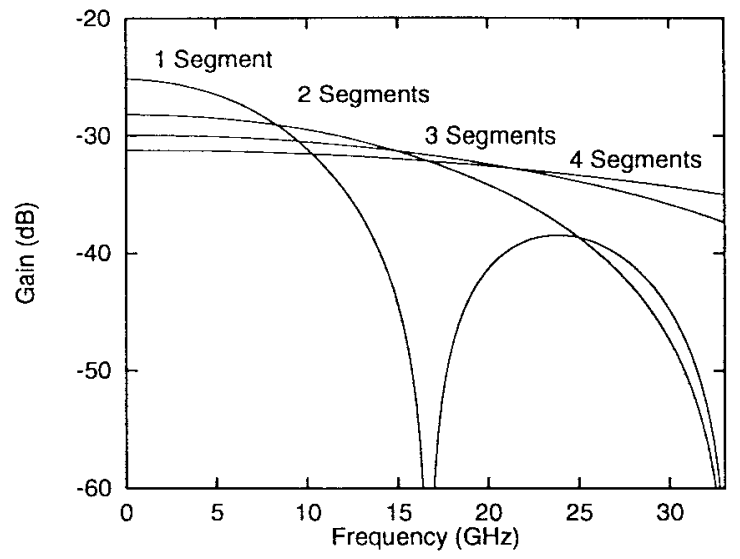

Fig. 15. The gain of a standard Mach-Zehnder with multiple electrode segments.

The results for the DCM2P superoctave modulator remain discouraging, even with rephasing. Fig. 14 shows the dynamic range from one-to-eight rephased electrode segments. This plot spans $2 \mathrm{GHz}$ only, which is enough to capture the interesting range. Even with eight electrodes, the frequency at which the dynamic range improvement over the simple MZM has fallen $10 \mathrm{~dB}$ is only $1.3 \mathrm{GHz}$. While this plot assumes an unrealistically low $1 \mathrm{~Hz}$ noise bandwidth, it also assumes perfect bias control. Given the modulators sensitivity to its three bias voltages, it is unlikely that this modulator is suitable for microwave applications with reasonable bandwidth.

Segmenting the RF electrode does have a low-frequency gain penalty. While flattening the gain versus bandwidth curve, it also lowers the absolute gain at low frequencies. The reduction occurs from the splitting of the RF power. It is split $N$ ways to feed the $N$ electrodes, reducing the voltage by $\sqrt{N}$ on each electrode segment. However, if the rephasing is achieved by a series intermittent interaction method such as described in [12], then there is no power splitting and subsequently no $1 / \sqrt{N}$ penalty. But this scheme involves long, curved, transmission lines and thus may have unacceptable losses. Fig. 15 shows the calculated gain of a simple Mach-Zehnder with one-, two-, three-, and four-electrode segments. Above some cross-over frequency each curve has a better absolute gain then the curve with one fewer electrode segments. Clearly, true velocity matching, by somehow making $\Delta n \rightarrow 0$ is preferable if possible, since there is no $\sqrt{N}$ penalty.

We wish to remind the reader that all the results obtained in this study may be applied to modulators with any degree of velocity matching by rescaling the frequency axis by the change in $\gamma$, the frequency-length-index product.

\section{ACKNOWLEDGMENT}

The authors would like to thank E. Ackerman, G. E. Betts, and C. H. Cox III of Lincoln Laboratories, Cambridge, MA, N. P. Bernstein of the Air Force Rome Laboratory, and J. H. Schaffner of Hughes Research Laboratories. The authors also wish to acknowledge the support and enthusiasm of the late B. Hendrickson of Rome Laboratories and ARPA.

\section{REFERENCES}

[1] W. B. Bridges and J. H. Schaffner, "Distortion in linearized electrooptic modulators," IEEE Trans. Microwave Theory and Tech., vol. 43, pp. 2184-2197, Sept. 1995.

[2] M. L. Farwell and W. S. C. Chang, "Simulating the response of coupled channel and interferometric modulator designs," J. Lightwave Technol., vol. 13, Oct. 1995

[3] B. Pucel, L. Riviere, and J. L. Bihan, "New model for the active directional coupler," J. Lightwave Technol., vol. 14, pp. 1501-1506, June 1996

[4] S. K. Korotky and R. M. Ridder, "Dual parallel modulation schemes for low-distortion analog optical transmission," IEEE J. Select. Areas Commun., vol. 8, pp. 1377-1381, Sept. 1990.

[5] M. L. Farwell, Z. Q. Lin, E. Wooten, and W. S. C. Chang, "An electrooptic intensity modulator with improved linearity," IEEE Photon. Technol. Lett., vol. 3, pp. 792-795, Sept. 1991.

[6] M. L. Farwell, W. S. C. Chang, and J. H. Schaffner, "A directional coupler modulator with improved linearity (operating up to $1 \mathrm{GHz}$ )," in Leos Summer Topical Meet. Dig. Broadband Analog and Digital Optoelectron., 1992, vol. WA3, pp. 8-9.

[7] G. E. Betts and F. J. O'Donnell, "Microwave analog optical links using suboctave linearized modulators," IEEE Photon. Technol. Lett., vol. 8, pp. 1273-1275, Sept. 1996.

[8] G. E. Betts, "Linearized modulator for suboctave-bandpass optical analog links," IEEE Trans. Microwave Theory Tech., vol. 42, pp. 2642-2649, Dec. 1994.

[9] H. Skeie and R. V. Johnson, "Linearization of electrooptic modulators by a cascade coupling of phase modulating electrodes," in Proc. SPIE, 1991, vol. 1583 , pp. 153-164.

[10] W. B. Bridges, F. T. Sheehy, and J. H. Schaffner, "Wave-coupled electrooptic modulators for microwave and millimeter-wave modulation," IEEE Photon. Technol. Lett., vol. 3, pp. 133-135, Feb. 1991.

[11] R. C. Alferness, S. K. Korotky, and E. A. J. Marcatili, "Velocitymatching techniques for integrated optic traveling wave switch modulators," IEEE J. Quantum. Electron., vol. QE-20, pp. 301-309, Mar. 1984.

[12] J. H. Schaffner, "Analysis of a millimeter wave integrated electro-optic modulator with a periodic electrode," in Proc. SPIE OE-LASE Conf. 1217, Los Angeles, CA, Jan. 16-17, 1990, pp. 101-110.

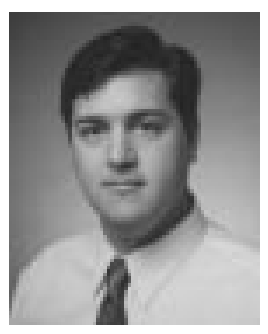

Uri V. Cummings was born in Vallejo, CA, on November 9, 1971. He received the B.A. degree in english from Wesleyan University, Middletown, $\mathrm{CT}$, and the B.S. degree from the California Institute of Technology (Caltech), Pasadena, simultaneously in 1994. He received the M.S. degree in electrical engineering from the Caltech in 1995. Currently, he is working toward the Ph.D. degree in electrical engineering at Caltech.

His research interests include the study of linearized electrooptic modulators and the design and experimental verification of antenna-arrayed electrooptic modulators at 100 $\mathrm{GHz}$. 


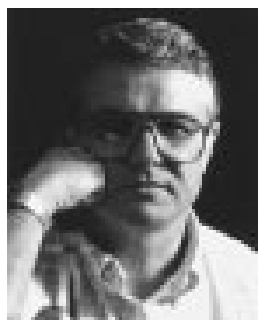

William B. Bridges (S'53-M'61-F'70-LF'98) was born in lnglewood, CA, in 1934. He received the B.S., M.S., and Ph.D. degrees in electrical engineering from the University of California, Berkeley, in 1956, 1957, and 1962, respectively. His graduate research dealt with noise in microwave tubes and electron-stream instabilities (which later became the basis of the Vircator.)

From 1957 to 1959, he was an Associate Professor in the Department of Electrical Engineering at the University of California, Berkeley, teaching courses in communication and circuits. Summer jobs at RCA and Varian provided stimulating experience with microwave radar systems, ammonia beam masers, and the early development of the ion vacuum pump. In 1960 , he joined the Hughes Research Laboratories, Malibu, CA, as a Member of the Technical Staff, and from 1968 to 1977, he was a Senior Scientist with a brief tour as Manager of the Laser Department in 1969-1970. His research at Hughes Research Laboratories involved gas lasers of all types and their application to optical communication, radar, and imaging systems. $\mathrm{He}$ is the discoverer of laser oscillation in noble gas ions and spent several years on the engineering development of practical high-power visible and ultraviolet ion lasers for military applications. He joined the faculty of the California Institute of Technology, Pasadena, CA, in 1977 as Professor of Electrical Engineering and Applied Physics, and serving as Executive Officer for Electrical Engineering from 1979 to 1981. In 1983, he was appointed Carl F. Braun Professor of Engineering and conducted research in optical and millimeter-wave devices and their applications. Current studies include the millimeter-wave modulation of light and high-fidelity analog microwave photonic links. He is coauthor (with C. K. Birdsall) of Electron Dynamics of Diode Regions (New York: Academic, 1966.)

Dr. Bridges is a member of Eta Kappa Nu, Tau Beta Pi, Phi Beta Kappa, and Sigma Xi, receiving Honorable Mention from Eta Kappa Nu as an "Outstanding Young Electrical Engineer" in 1966. He received the Distinguished Teaching Award in 1980 and 1982 from the Associated Students of Caltech, the Arthur L. Schawlow Medal from the Laser Institute of America in 1986, and the IEEE LEOS Quantum Electronics Award in 1988. He is a member of the National Academy of Engineering and the National Academy of Sciences, and a Fellow of the Optical Society of America (OSA) and the Laser Institute of America. He was a Sherman Fairchild Distinguished Scholar at Caltech, Pasadena, CA, in 1974-1975, and a Visiting Professor at Chalmers Technical University, Göteborg, Sweden, in 1989. He has served on various committees of both IEEE and OSA and was formerly Associate Editor of the IEEE Journal of QuANTUM Electronics and the Journal of the Optical Society of America. He was the President of the Optical Society of America in 1988, a member of the United States Air Force Scientific Advisory Board 1985-1989, and a member of the Board of Directors of Uniphase Corporation 1986-1998. 